\title{
GROUPS WITH MANY PRONORMAL SUBGROUPS
}

\author{
MARIA FERRARA $\left(\mathbb{D}\right.$ and MARCO TROMBETTI $\mathbb{1}^{\otimes}$
}

(Received 21 February 2021; accepted 10 March 2021; first published online 11 May 2021)

\begin{abstract}
A subgroup $H$ of a group $G$ is pronormal in $G$ if each of its conjugates $H^{g}$ in $G$ is conjugate to it in the subgroup $\left\langle H, H^{g}\right\rangle$; a group is prohamiltonian if all of its nonabelian subgroups are pronormal. The aim of the paper is to show that a locally soluble group of (regular) cardinality in which all proper uncountable subgroups are prohamiltonian is prohamiltonian. In order to obtain this result, it is proved that the class of prohamiltonian groups is detectable from the behaviour of countable subgroups. Examples are exhibited to show that there are uncountable prohamiltonian groups that do not behave very well. Finally, it is shown that prohamiltonicity can sometimes be detected through the analysis of the finite homomorphic images of a group.
\end{abstract}

2020 Mathematics subject classification: primary 20E15; secondary 20E25, $20 \mathrm{~F} 16$.

Keywords and phrases: prohamiltonian group, pronormal subgroup, countably recognisable group class.

\section{Introduction}

Let $G$ be a group. A subgroup $H$ of $G$ is said to be pronormal in $G$ if $H$ and $H^{g}$ are conjugate in $\left\langle H, H^{g}\right\rangle$ for any $g \in G$. Pronormal subgroups were introduced by P. Hall as a wide generalisation of many subgroup properties. It is in fact clear that any normal subgroup and any maximal subgroup is pronormal; moreover, this is also the case for Sylow $p$-subgroups of finite groups and Hall $\pi$-subgroups of finite soluble groups. The first nontrivial results on pronormality were obtained by Rose in [29] and, more recently, several authors have investigated the behaviour of groups with many pronormal subgroups. Peng [24] showed that for finite groups, having only pronormal subgroups is precisely the same as being soluble T-groups (that is, soluble groups in which normality is a transitive relation) and this result was later extended from finite groups to FC-groups, that is, groups in which every element has only finitely many conjugates (see [11]). Since a soluble T-group is metabelian and transitivity of normality is a local property (see [28]), the result of Peng shows also that a locally finite group with only pronormal subgroups is a metabelian T-group. Actually, all these

The authors are supported by GNSAGA (INdAM) and are members of the non-profit association 'Advances in Group Theory and Applications' (www.advgrouptheory.com). The first author is supported by the project 'Groups: overlappings between Algebra and Geometry, Logic and Mathematics Education' (VALERE: VAnviteLli pEr la RicErca).

(C) Australian Mathematical Publishing Association Inc. 2021. This is an Open Access article, distributed under the terms of the Creative Commons Attribution licence (http://creativecommons.org/ licenses/by/4.0/), which permits unrestricted re-use, distribution, and reproduction in any medium, provided the original work is properly cited. 
results are very special cases of a recent theorem showing that a locally graded group with only pronormal subgroups is metabelian (see, for instance, [10, Lemma 2.8]); a group $G$ is said to be locally graded if every finitely generated nontrivial subgroup of $G$ contains a proper subgroup of finite index.

The study of groups whose nonabelian subgroups satisfy a strong subgroup theoretical property, such as normality, has been a very flourishing subject in both finite and infinite group theory. The description of such groups can sometimes be very precise and may encompass many group classes such as minimal nonabelian groups and Dedekind groups, providing a very useful tool in studying arbitrary groups. The structure of metahamiltonian groups, that is, groups whose nonnormal subgroups are abelian, is nowadays well understood, especially in the soluble case (see, for instance, [5]). Recently, the study of (soluble) prohamiltonian groups, that is, groups whose nonabelian subgroups are pronormal, has started and the first relevant structural results may be found in [2, 3]; of course, all metahamiltonian groups and all groups with only pronormal subgroups are prohamiltonian. The fact that such a class is much bigger than that of metahamiltonian groups may be seen from the following facts:

(1) soluble prohamiltonian groups have derived length at most four and there are plenty of examples with derived lengths three and four (these are essentially completely characterised in [2]);

(2) there exist finite simple groups (and even sporadic groups) that are prohamiltonian (see [3, 19]).

The aim of this paper is to give a further impulse to the newborn theory of prohamiltonian groups showing that prohamiltonicity can be recognised through the analysis of certain large classes of subgroups. Thus, in order to check if a 'large' group is prohamiltonian or not, one just needs to focus on some smaller pieces of the group; such results will be useful in the proof of sufficiency in certain characterisations of prohamiltonian groups.

Our first main theorem shows that if all countable subgroups of a group are prohamiltonian, then the group itself must be such.

THEOREM 1.1. The class of prohamiltonian groups is countably recognisable.

In order to prove this theorem, we do not take into account the structure of soluble prohamiltonian groups described in [2]. On the other hand, we will use this description in combination with Theorem 1.1 to obtain a much deeper result.

THEOREM 1.2. Let $\boldsymbol{\aleph}$ be a cardinal with cofinality $\operatorname{cf}(\boldsymbol{\aleph})>\boldsymbol{\aleph}_{0}$ and let $G$ be a locally soluble group of cardinality $\boldsymbol{\aleph}$. If all proper subgroups of $G$ of cardinality $\boldsymbol{\aleph}$ are prohamiltonian, then $G$ is prohamiltonian.

In fact, we prove a more general statement than Theorem 1.2 (see Theorem 2.3) that allows us to replace local solubility by many other group classes. It also turns out that if one assumes the generalised continuum hypothesis $(\mathrm{GCH})$, then the assumption on the cofinality can be dropped. 
THEOREM 1.3. Assume that the GCH holds and let $\mathbf{\aleph}$ be an uncountable cardinal. If $G$ is a locally soluble group of cardinality $\boldsymbol{\aleph}$ whose proper subgroups of cardinality $\boldsymbol{\aleph}$ are prohamiltonian, then $G$ is prohamiltonian.

In the last part of the paper we prove that, as for many other natural classes of groups (such as nilpotency), the class of prohamiltonian groups can be recognised in some circumstances through the analysis of the finite quotients.

THEOREM 1.4. Let $G$ be a finitely generated hyper(abelian or finite) group whose finite homomorphic images are prohamiltonian. Then $G$ is prohamiltonian.

Most of our notation is standard and can be found in [26].

\section{The countable character}

A group class $X$ is said to be countably recognisable if, whenever all countable subgroups of a group $G$ belong to $X$, then $G$ itself is an $X$-group. Countably recognisable classes of groups were introduced by Baer in [1] as a tool for studying 'large' (that is, uncountable) groups through their 'small' (that is, countable) subgroups. In his wake, many other group classes were shown to be countably recognisable (see, for instance, $[13,16,8]$ ), a fact leading to a better understanding of uncountable groups with restrictions on large subgroups (see, for example, [18, Theorem 20] and the papers $[6,7])$. In particular, it has recently been proved in [10] that a subgroup is pronormal in $G$ if and only if all of its countable subgroups are pronormal; of course, this fact immediately implies that the class of groups with only pronormal subgroups is countably recognisable. One of the aims of this section is to extend these results showing that pronormality of a nonabelian subgroup can be recognised only by the behaviour of the nonabelian countable subgroups.

Before proving the first result of the section, we quote the following easy lemma (we refer to [10] for a proof) showing that if $X$ is a pronormal subgroup of a group $G$, then $X^{g} \leq X$ is equivalent to $X=X^{g}$ for all $g \in G$; using the terminology introduced in [10], any pronormal subgroup is weakly pronormal.

LEMMA 2.1. Let $G$ be a group and let $X$ be a subgroup of $G$ such that $X^{g}$ is contained in $N_{G}(X)$ for some element $g$ of $G$. If $X$ and $X^{g}$ are conjugate in $\left\langle X, X^{g}\right\rangle$, then $X^{g}=X$.

THEOREM 2.2. Let $G$ be a group and let $X$ be a nonabelian subgroup of $G$. If all countable nonabelian subgroups of $X$ are pronormal in $G$, then $X$ itself is pronormal in $G$.

PROOF. Assume for a contradiction that $X$ is not pronormal in $G$, so that there exists an element $g$ of $G$ such that $X$ and $X^{g}$ are not conjugate in $\left\langle X, X^{g}\right\rangle$. Let $a$ be any element of $\left\langle X, X^{g}\right\rangle$ such that $X^{a}$ is (strictly) contained in $X^{g}$. Thus, $X^{h}<X$ for $h=a g^{-1}$. Moreover, since $X$ is a nonabelian subgroup generated by the set $X \backslash X^{h}$, it is possible to find noncommuting elements $x$ and $y$ in $X \backslash X^{h}$. Now, the countable nonabelian subgroup

$$
Z=\left\langle x, y, x^{h}, y^{h}, \ldots, x^{h^{n}}, y^{h^{n}}, \ldots\right\rangle
$$


is pronormal in $G$ and, of course, $Z^{h} \leq Z$. It follows from Lemma 2.1 that $Z=Z^{h} \leq X^{h}$ and this contradicts the fact that both $x$ and $y$ do not belong to $X^{h}$. Consequently, for all $a \in\left\langle X, X^{g}\right\rangle$, there is an element $x(a)$ of $X$ such that $x(a)^{a}$ does not belong to $X^{g}$.

Now, since $X$ is nonabelian, it must contain two noncommuting elements $x$ and $y$. Let $Z_{0}=\langle x, y\rangle$ and suppose that we have already defined $Z_{n}$ for some nonnegative integer $n$. Then we put

$$
Z_{n+1}=\left\langle Z_{n}, x(a): a \in\left\langle Z_{n}, Z_{n}^{g}\right\rangle\right\rangle
$$

and recursion proceeds. Thus, the subgroup $Z_{m}$ is defined for all nonnegative integers $m$. It is clear that all of these nonabelian subgroups are countable and that their union $Z$ is nonabelian and countable as well. Therefore, $Z$ is pronormal in $G$ and so there exists an element $b \in\left\langle Z, Z^{g}\right\rangle$ such that $Z^{b}=Z^{g}$. Hence, there is a nonnegative integer $m$ such that $b$ belongs to $\left\langle Z_{m}, Z_{m}^{g}\right\rangle$, which means that $x(b)$ is an element of $Z_{m+1} \leq Z$. However, this immediately gives rise to the contradiction $x(b)^{b} \in Z^{b}=Z^{g} \leq X^{g}$. The theorem is proved.

We are now in a position to prove our first main theorem.

Proof of Theorem 1.1. Let $G$ be a group and suppose that all of its countable subgroups are prohamiltonian. Let $X$ be any nonabelian subgroup of $G$ and consider a nonabelian countable subgroup $H$ of $X$. It is easy to see that $H$ is pronormal in $G$ : if $g$ is any element of $G$, then $H$ is a nonabelian subgroup of the countable prohamiltonian subgroup $\langle H, g\rangle$ and hence there exists an $x \in\left\langle H, H^{g}\right\rangle$ such that $H^{x}=H^{g}$. We can now apply Theorem 2.2 in order to obtain the pronormality of $G$.

In contrast to Theorems 2.2 and 1.1, prohamiltonicity cannot be recognised from the behaviour of finitely generated subgroups, as the following example shows. Let $X_{0}$ be any nonabelian group of order 21 and let $\left\{p_{n}: n \in \mathbb{N}\right\}$ be any sequence of prime numbers all greater than seven. For $n \geq 1$, put

$$
X_{n}=\left\langle a_{n} b_{n}: a_{n}^{2}=b_{n}^{p_{n}}=1, a_{n} b_{n}=b_{n}^{-1} a_{n}\right\rangle
$$

and in the cartesian product of all of the groups $X_{n}$ (with $n \geq 0$ ) consider the subgroup

$$
G=\left\langle a, \operatorname{Dr}_{n \in \mathbb{N}_{0}} X_{n}\right\rangle,
$$

where $a$ is such that $a(0)=1$ and $a(n)=a_{n}$ for all $n \in \mathbb{N}$. Thus, $G$ is a locally finite $\overline{\mathrm{T}}$-group (see, for instance, [28, Theorem 6.1.1]), so that in particular all of its finite subgroups are pronormal. On the other hand, conjugation by $a$ shows that the nonabelian subgroup

$$
X_{0} \times\left\langle a_{n} b_{n}: n \in \mathbb{N}\right\rangle
$$

is not pronormal in $G$.

We notice that there exist large (in the sense of cardinality) prohamiltonian groups. In fact, Ehrenfeucht and Faber [14] constructed uncountable extraspecial $p$-groups in which all abelian subgroups are countable; on the other hand, using the 
Ol'shanskii machinery, it is possible to construct simple prohamiltonian groups of uncountable cardinality satisfying the minimal condition on subgroups, as follows. Let $X$ be a group with no involutions and satisfying the minimal condition on subgroups, so in particular without proper isomorphic subgroups, and, using Theorem 35.1 of [22], embed $X$ in a countable simple group $G_{X}$ such that:

(i) every proper subgroup of $G_{X}$ is either cyclic of odd order or conjugate to a subgroup of $X$;

(ii) $G_{X}=\langle x, y\rangle$ for all $x, y \in G_{X}$ such that $x \in X$ and $y \notin X$.

Start by taking $X=\mathbb{Z}_{3}$ and $G_{0}=G_{X}$. Suppose that we have already defined $G_{\lambda}$ for all countable ordinals $\lambda<\alpha$ and put

$$
G_{\alpha}= \begin{cases}G_{G_{\lambda}} & \text { if } \alpha=\lambda+1, \\ \bigcup_{\lambda<\alpha} G_{\lambda} & \text { otherwise. }\end{cases}
$$

Finally, let $G=G_{\aleph_{1}}$ be the union of the $G_{\lambda}$ with $\lambda<\boldsymbol{\aleph}_{1}$. It is not difficult to see that $G$ has cardinality $\boldsymbol{\aleph}_{1}$ and that any proper subgroup of $G_{\lambda}$, for some nonzero limit ordinal $\lambda$, is contained in a $G_{\kappa}$ for some countable successor ordinal $\kappa<\lambda$ (see the proof of Theorem 35.2 of [22]); in particular, all proper subgroups of $G$ are countable. In fact, it is easy to see that for each ordinal $\alpha$ all nonabelian proper subgroups of $G_{\alpha}$ are conjugate to a $G_{\lambda}$ for some $\lambda<\alpha$; moreover, $G_{\lambda_{1}}$ is not isomorphic to $G_{\lambda_{2}}$ whenever $\lambda_{1} \neq \lambda_{2}$. Therefore, if two nonabelian subgroups of $G_{\alpha}$ are isomorphic, then they must also be conjugate.

Now, let $H$ be any nonabelian proper subgroup of $G$ and let $g \in G \backslash H$. From the previous remarks, $K=\left\langle H, H^{g}\right\rangle$ is isomorphic to a $G_{\kappa}$ for some ordinal $\kappa$, so that all isomorphic nonabelian subgroups of $K$ must be conjugate; in particular, $H$ is conjugate to $H^{g}$ in $K$, which is what we wanted to prove. Therefore, $G$ is prohamiltonian and satisfies the required properties.

It was proved in [9] that the commutator subgroup of an arbitrary metahamiltonian group is always 2-generated and in particular it is countable. The previous example shows that this is very far from being true for arbitrary prohamiltonian groups.

It should also be pointed out that it is not clear if an approach similar to the one above may be used to obtain uncountable simple groups with only pronormal subgroups: indeed, at any step one could have isomorphic cyclic subgroups which are not conjugate.

Finally, using Theorem 1.1 and the structure of soluble prohamiltonian groups described in [2], we prove that generalised soluble uncountable groups whose proper large subgroups are prohamiltonian must often be themselves prohamiltonian.

THEOREM 2.3. Let $\boldsymbol{\aleph}$ be a cardinal with cofinality $\operatorname{cf}(\boldsymbol{\aleph})>\boldsymbol{\aleph}_{0}$ and let $G$ be a locally graded group of cardinality $\mathbf{\aleph}$ with no simple homomorphic image of cardinality $\mathbf{\aleph}$. If all proper subgroups of $G$ of cardinality $\boldsymbol{\aleph}$ are soluble-by-finite and prohamiltonian, then $G$ is soluble-by-finite and prohamiltonian. 
Proof. Suppose first that $G$ is not soluble-by-finite; in particular, $G$ has no proper subgroup of finite index. Let $N$ be a proper normal subgroup of $G$ of cardinality $\boldsymbol{\aleph}$. Then $N$ is a soluble-by-finite prohamiltonian group and it follows from [21] that $G / N$ is still locally graded, so it cannot be finitely generated. If $X / N$ is a proper subgroup of $G / N$, then $X$ is a proper subgroup of $G$ and hence it is soluble-by-finite. Thus, $G / N$ is a locally (soluble-by-finite) group with all proper subgroups soluble-by-finite. It follows from [12] that if $G / N$ is not locally soluble, then it is either soluble-by-PSL $(2, F)$ or soluble-by-Sz $(F)$ for some infinite locally finite field $F$. However, those simple groups contain arbitrarily large, nested finite simple groups of the same types, contradicting the main result of [3]. Thus, $G / N$ is locally soluble (of derived length at most four) and hence it is even soluble, which is a contradiction. Thus, all proper normal subgroups of $G$ have cardinality $<\boldsymbol{N}$. In this case, if $N$ is any proper normal subgroup of $G$, then $G / N$ contains a proper subgroup $X / N$ of cardinality $\boldsymbol{\aleph}$ (see, for instance, Corollary 2.6 of [6]) and hence $N<X$ is a soluble-by-finite prohamiltonian group. Let $M$ be the product of all soluble normal subgroups of $G$, which is easily seen to be soluble of derived length at most four, implying that $|G / M|=\aleph$. Of course, all proper normal subgroups of $G / M$ are finite and so even central in $G / M$. Since $G$ has no simple homomorphic image of cardinality $\boldsymbol{\aleph}$ and all proper normal subgroups are of cardinality $<\boldsymbol{\aleph}$, it follows that $G / M$ is the product of all of its proper normal subgroups and hence it must be abelian. This last contradiction shows that $G$ is actually soluble-by-finite.

Let $E$ be any countable subgroup of $G$. We now prove that if $G$ contains a normal abelian subgroup $A$ of cardinality $\boldsymbol{\aleph}$, then $E$ is contained in a proper subgroup of cardinality $\boldsymbol{\kappa}$. Of course, we may assume that $G=E A$, so that $A \cap E$ is a countable normal subgroup of $G$ and hence we may even assume that $A \cap E=1$; for similar reasons, it is also possible to assume that $C_{E}(A)=1$. This shows that all proper subgroups of $G / A \simeq E$ have only pronormal subgroups and so $E$ is either finite or has only pronormal subgroups itself by Theorem 2.11 of [10].

Assume first that $G$ is metabelian. Then either $G / G^{\prime}$ or $G^{\prime}$ have cardinality $\mathbf{\aleph}$. In the former case, we may easily find a normal subgroup $N$ such that both $N$ and $G / N$ have cardinality $\boldsymbol{N}$ (see [6, Lemma 2.4]); thus, $E<E N<G$, proving the claim. In the latter case (assuming that $\left|G / G^{\prime}\right|<\boldsymbol{\aleph}$ ), we can use Proposition 3 of [23] to find a proper $G$-invariant subgroup $A_{1}$ of $A$ of cardinality $\boldsymbol{\aleph}$; again $E<E A_{1}<G$, proving the claim. Therefore, $G$ can be assumed nonmetabelian; in particular, $E$ is nonabelian.

If $E$ is finite, using Lemma 2.3 of [6], we can find a proper $E$-invariant subgroup in $A$ of cardinality $\mathbf{\aleph}$, again proving the claim. Thus, $E$ has only pronormal subgroups and so it is metabelian and periodic (see [20]); in particular, $E$ is not finitely generated and $G$ has derived length three.

If $G^{\prime \prime}<A$, then $G^{\prime \prime}$ has cardinality $<\boldsymbol{\aleph}$ and $\left|G / G^{\prime \prime}\right|=\boldsymbol{\aleph}$. Thus, the claim holds for $G / G^{\prime \prime}$ and $E G^{\prime \prime}$ is contained in a proper subgroup of $G$ of cardinality $\aleph$. So, it is possible to assume that $G^{\prime \prime}=A$. Now, any finitely generated subgroup of $G$ is contained in a finitely generated prohamiltonian subgroup of derived length three and so is periodic (see Theorem 2.5 of [2]). It follows that $G$ is periodic, so, if we assume by 
contradiction that $A$ contains no proper $G$-invariant subgroup of cardinality $\aleph$, then $A$ must be an abelian $p$-group for some prime $p$. Moreover, from the assumptions on $E$ we may easily see that no $p^{\prime}$-element centralises $A$.

Thus,

$$
G=\bigcup_{i \in \mathbb{N}} X_{i}
$$

is the countable union of a chain of prohamiltonian subgroups $X_{i}$ containing $A$ and such that $X_{i}^{\prime \prime}$ has order greater than $i$. Using Theorem 3.8 of [2], we may assume that the order of $X_{i}^{\prime \prime}$ is finite and that $\mathrm{O}_{p}\left(X_{i}\right)$ is abelian for all $i$, so it must coincide with $A$. Moreover, it follows from Theorem 3.8( $\left.\mathrm{c}_{\mathrm{ii}}\right)_{2}$ of [2] that $A$ is a Sylow $p$-subgroup of each $X_{i}$. For each $i$, one can therefore write $A=X_{i}^{\prime \prime} \times Z\left(X_{i}^{\prime}\right) \times Z\left(X_{i}\right)$ by Theorem 3.8 $\left(\mathrm{b}_{\mathrm{ii}}\right)_{1}$ of [2]; but this yields a contradiction in $X_{i+1}$ by $\left(\mathrm{a}_{\mathrm{ii}}\right),\left(\mathrm{b}_{\mathrm{ii}}\right)_{2}$ and $\left(\mathrm{b}_{\mathrm{ii}}\right)_{3}$ of Theorem 3.8 of [2]. This contradiction proves the claim and shows that $E$ is contained in a proper subgroup of cardinality $\boldsymbol{\aleph}$ whenever $G$ contains an abelian normal subgroup of cardinality $\boldsymbol{\aleph}$.

Let $S$ be the soluble radical of $G$ and let $H$ be the first term of the derived series of $S$ with cardinality $<\boldsymbol{\aleph}$. Then $G / H$ contains a normal abelian subgroup of cardinality $\mathbf{\aleph}$. Using what we have just proved, $H E$ is contained in a proper subgroup of cardinality $\aleph$. Therefore, all countable subgroups of $G$ are prohamiltonian and Theorem 1.1 completes the proof.

Theorem 1.2 follows from the previous result using Corollary 2.6 of [6] and the fact that soluble prohamiltonian groups have derived length less than or equal to four.

Assuming the $\mathrm{GCH}$, the condition on the cofinality of the cardinal number $\boldsymbol{\aleph}$ can be dropped. Indeed, the fact that $\operatorname{cf}(\boldsymbol{N})>\boldsymbol{\aleph}_{0}$ is used in the proof of Theorem 2.3 only through an application of Proposition 3 of [23]. If we use Corollary 4 of [23] instead of Proposition 3, we obtain a proof of Theorem 2.3 (and so of Theorem 1.3) not requiring the condition on the cofinality.

We conclude this section with some remarks. First of all, we notice that the proof of Theorem 2.3 yields something more if one assumes the existence of large abelian normal subgroups.

THEOREM 2.4. Let $\boldsymbol{\aleph}$ be a cardinal with cofinality $\operatorname{cf}(\boldsymbol{\aleph})>\boldsymbol{\aleph}_{0}$ and let $G$ be a group of cardinality $\boldsymbol{\aleph}$ whose proper subgroups of cardinality $\mathbf{N}$ are soluble-by-finite and prohamiltonian. If $\mathbf{G}$ contains a proper abelian normal subgroup of cardinality $\mathbf{\aleph}$, then every proper countable subgroup of $G$ is contained in a proper subgroup of cardinality $\boldsymbol{N}$.

Here pronormality seems to be essential, but the hypothesis on the cofinality may be removed as before.

Secondly, in the next section we prove that prohamiltonian groups are often soluble-by-finite. This is the case, for instance, when they are hyper(abelian or finite), FC-groups or locally finite. It easily follows from Corollary 2.6 of [6] that a group of uncountable cardinality $\boldsymbol{\aleph}$ that is either hyper(abelian or finite), or an FC-group, contains a proper subgroup of cardinality $\mathbf{\aleph}$. Thus, we get a result analogous 
to Theorem 1.2 (and Theorem 1.3) if we replace in it 'locally soluble' by 'hyper(abelian or finite)' or 'FC'.

For locally finite groups we cannot use Corollary 2.6 of [6], but it is known that an uncountable locally finite group of regular cardinality has a proper subgroup of the same cardinality (see, for instance, [6, page 387]). Since this condition is stronger than that on the cofinality, it turns out that for locally finite groups we get a 'weaker' version of Theorem 1.2 if we replace 'a cardinal with cofinality $\operatorname{cf}(\boldsymbol{\aleph})>\boldsymbol{\aleph}_{0}$ ' by 'an uncountable regular cardinal'.

Furthermore, as we will shortly see in the next section, Corollary 3.3 shows that any locally (soluble-by-finite) prohamiltonian group is actually soluble-by-finite. Thus, one could restate Theorem 1.2 (and Theorem 1.3) for a locally (soluble-by-finite) group and remove the local graduation and the solubility assumptions on the large subgroups.

Thirdly, the argument in the first part of the proof of Theorem 2.3 shows that a locally graded group whose proper subgroups are soluble-by-finite prohamiltonian must itself be soluble-by-finite and hence either abelian-by-finite or soluble.

\section{Prohamiltonian finite homomorphic images}

Within the universe of finitely generated soluble groups, certain group-theoretical properties can be detected from the behaviour of finite homomorphic images. Nilpotency is probably the most remarkable of these properties (see [25]), but many other group classes behave similarly (see, for instance, $[4,15,17])$. The aim of this section is to show that prohamiltonicity has the same good behaviour.

Before proving the main result of this section, we need to show that in many circumstances generalised soluble prohamiltonian groups are soluble-by-finite. Unfortunately, it seems to be a much more difficult task to understand if a locally graded prohamiltonian group should be soluble-by-finite or not; in large measure, this is because we do not know if there exist locally graded simple groups which are prohamiltonian.

THEOREM 3.1. Let $G$ be a group admitting a normal series of subgroups with either locally soluble or locally finite factors. If $G$ is prohamiltonian, then $G$ is soluble-by-finite.

ProOF. Suppose first that $G$ is hyper(abelian or finite), that is, $G$ admits an ascending normal series

$$
\{1\}=G_{0} \leq G_{1} \leq \cdots \leq G_{\gamma} \leq G_{\gamma+1} \leq \cdots \leq G_{\alpha}=G
$$

whose factors are either normal or finite. We begin by proving that every infinite homomorphic image of $G$ contains a nontrivial abelian normal subgroup: it is easy to see that it suffices to prove such a claim on $G$ with $\alpha=\omega$ and all factors $G_{i+1} / G_{i}$ finite and nonabelian. Since $G_{1}$ is nonabelian, $G / G_{1}$ has only pronormal subgroups. Thus, by the consequence of Peng's theorem mentioned in Section 1, it follows that $G / G_{1}$ is metabelian. Since $G_{1}$ is finite, $G$ is soluble-by-finite and hence contains a 
nontrivial abelian normal subgroup, proving the claim. What we have just proved implies that $G$ contains a subgroup of finite index that is hyperabelian, so we may assume that $G$ itself is hyperabelian; in particular, all factors $G_{\gamma+1} / G_{\gamma}$ are abelian. If any, let $\lambda$ be the smallest ordinal such that $X=G_{\lambda}$ is not soluble. It was proved in [2] that soluble prohamiltonian groups have derived length at most four and this clearly means that $\lambda$ cannot be a limit ordinal. For, otherwise, $G_{\lambda}$ is locally soluble of derived length at most four and so soluble of derived length at most four. However, $\lambda$ cannot be a successor ordinal either, since otherwise $G_{\lambda-1}$ would be soluble together with $G_{\lambda}$. Therefore, $G=G_{\alpha}$ is soluble and the statement is proved in the hyper(abelian or finite) case.

Suppose now that $G$ is locally finite and not soluble-by-finite. Since any locally nilpotent prohamiltonian group is metahamiltonian and hence soluble-by-finite, the previous case allows us to assume that $G$ has a trivial Hirsch-Plotkin radical. However, it has been proved in [3] that an infinite locally finite prohamiltonian group cannot be simple; thus, the infinite subgroup $G^{(i v)}$ contains a proper nontrivial normal subgroup $N$ that cannot be abelian. Now, $G^{(i v)} / N$ is metabelian since all of its subgroups are pronormal and hence $G / G^{(v)}$ has derived length five, contradicting Lemma 2.1 of [2].

We turn now to the general case and using what has been proved so far we may assume that $G$ admits a normal series of subgroups $\mathcal{N}$ with either abelian or finite factors. Let $M_{1}$ be the union of all terms of the normal series $\mathcal{N}$ that are abelian and let $M_{2}$ be the intersection of all terms that are not abelian. Since $\mathcal{N}$ is complete as a series, the subgroups $M_{1}$ and $M_{2}$ belong to it; moreover, $M_{1}$ is abelian, $G / M_{2}$ is metabelian (as each nonabelian subgroup $N$ of $\mathcal{N}$ is such that $G / N$ is locally graded with only pronormal subgroups) and $M_{2} / M_{1}$ is a (possibly trivial) factor of the normal series $\mathcal{N}$. Therefore, $G$ is soluble-by-finite and the theorem is proved.

COROLLARY 3.2. Let $G$ be a prohamiltonian group belonging to one of the following classes of groups: FC-groups, hyper(abelian or finite) groups and locally finite groups. Then $G$ is soluble-by-finite.

It is proved in [2, Corollary 2.4] that every finitely generated soluble prohamiltonian group has a finite commutator subgroup. The proof of the following corollary shows in particular that all finitely generated soluble-by-finite prohamiltonian groups have a finite commutator subgroup and generalises Theorem 3.1 to a wide range of group classes.

COROLlary 3.3. Let $G$ be a locally (soluble-by-finite) prohamiltonian group. Then $G$ is soluble-by-finite.

ProOF. Let $F$ be a finitely generated subgroup of $G$. Then there is a normal soluble subgroup $S$ of $F$ such that $|F: S|<\infty$; moreover, our previous remark shows that $S^{\prime}$ is finite. Suppose that $S$ is not periodic. Then the periodic part $T$ of $S$ is finite and in order to prove that $F^{\prime}$ is finite we can get rid of $S^{\prime} T$; in particular, we can assume that $S$ is torsion-free. Let $f$ be any element of $F$. Now $X=\langle f\rangle S$ is soluble finitely generated, 
so $X^{\prime}$ is finite and hence $X^{\prime}=1$. The arbitrariness of $f$ in $F$ shows that $S \leq Z(F)$ and, by the celebrated theorem of Schur, $F^{\prime}$ is finite, completing the proof of the claim.

The commutator subgroup $G^{\prime}$ of $G$ is now locally finite and Theorem 3.1 implies that $G^{\prime}$ is soluble-by-finite. Therefore, $G$ is soluble-by-finite and the statement is proved.

The following lemma will be very useful in the proof of Theorem 1.4.

LEMMA 3.4. Let $G$ be a residually finite group whose finite homomorphic images are prohamiltonian. Then every subgroup of finite index of $G$ is either abelian or pronormal. In particular, $G$ is either metabelian or abelian-by-finite.

ProOf. Let $X$ be a subgroup of $G$ of finite index that is not pronormal in $G$. Since $X$ has finite index in $G$ and $G$ is residually finite, there exists a family of $G$-invariant subgroups $\mathcal{N}=\left\{N_{i}\right\}_{i \in I}$ which are contained in $X$ and have trivial intersection. Let $N$ be any element of $\mathcal{N}$ and notice that $X / N$ cannot be pronormal in the finite factor group $G / N$. Thus, $X / N$ is abelian and the arbitrariness of $N$ in $\mathcal{N}$ shows that $X^{\prime}=\{1\}$.

Suppose now that $G$ is not abelian-by-finite. Thus, all subgroups of finite index are pronormal and in particular all finite homomorphic images are metabelian by Peng's theorem. Since this obviously implies that $G$ is metabelian, the statement is proved.

We are now in a position to prove our last main theorem.

Proof of Theorem 1.4. Assume for a contradiction that $G$ is not prohamiltonian. The remark before Corollary 3.3 shows that the class of all finitely generated, soluble-by-finite prohamiltonian groups consists of finitely presented groups. By Lemma 6.17 of [26], $G$ has an (infinite) homomorphic image which is not prohamiltonian but all of whose proper homomorphic images are prohamiltonian. By hypothesis, there exists a nontrivial normal subgroup $L$ which is either finite or abelian; as $G / L$ is prohamiltonian, it follows that $G$ is soluble-by-finite by Theorem 3.1. On the other hand, if $N$ is the smallest nontrivial term of the derived series of the soluble radical of $G$, then $G / N$ is abelian-by-finite by (the remark after) Corollary 2.4 of [2] and so $G$ is metabelian-by-finite; in particular, $G$ is residually finite by Theorem 9.51 of [26].

Now, by an application of Lemma 3.4, either $G$ contains an abelian subgroup of finite index or all subgroups of finite index of $G$ are pronormal; in the latter case, $G$ is even soluble by Peng's theorem and cannot be a T-group by Theorem 3.3.1 of [28]. Therefore, by Theorem 2 of [27], $G$ has a finite homomorphic image which is not a T-group, contradicting Peng's theorem. It follows that $G$ is abelian-by-finite and so polycyclic-by-finite.

Let $X$ be any nonabelian, nonpronormal subgroup of $G$; in particular, there are elements $x_{1}$ and $x_{2}$ of $X$ such that $\left[x_{1}, x_{2}\right] \neq 1$. Since $G$ is residually finite, we may find a normal subgroup $N$ of finite index not containing $\left[x_{1}, x_{2}\right]$. Now, let $M$ be any normal subgroup of $G$ of finite index. Then $X(M \cap N) /(M \cap N)$ is a nonabelian subgroup of the prohamiltonian group $G /(M \cap N)$ and so it is pronormal. It clearly follows that 
$X M / M$ is a pronormal subgroup of $G / M$. Thus, $X^{\sigma}$ is pronormal in $G^{\sigma}$ for every finite homomorphic image $G^{\sigma}$ of $G$ and hence $X$ is pronormal in $G$ (see Theorem 2.8 of [11]), which is a contradiction. The theorem is proved.

Notice that the previous theorem cannot be much improved. In fact, the consideration of any perfect locally nilpotent group (for instance McLain's characteristically simple groups described at page 14 of Part 2 of [26]) shows that a locally nilpotent group whose finite homomorphic images are prohamiltonian need not be prohamiltonian (and not even soluble-by-finite). Moreover, the Ol'shanskii machinery (see Section 2) can be easily employed to construct examples of infinite finitely generated simple groups which are not prohamiltonian.

Finally, we observe that the arguments in the proof of Theorem 1.4 can be used to prove a similar result concerning finitely generated groups whose finite homomorphic images have only pronormal subgroups.

THEOREM 3.5. Let $G$ be a finitely generated hyper(abelian or finite) group whose (nonabelian) subgroups of finite index are pronormal. Then all (nonabelian) subgroups of $G$ are pronormal.

\section{References}

[1] R. Baer, 'Abzählbar erkennbare gruppentheoretische Eigenschaften', Math. Z. 79 (1962), 344-363.

[2] M. Brescia, M. Ferrara and M. Trombetti, 'Groups whose subgroups are either abelian or pronormal', Preprint.

[3] M. Brescia and M. Trombetti, 'Locally finite simple groups whose non-abelian subgroups are pronormal', Preprint.

[4] M. De Falco, F. de Giovanni and C. Musella, 'Groups whose finite homomorphic images are metahamiltonian', Comm. Algebra 37 (2009), 2468-2476.

[5] M. De Falco, F. de Giovanni and C. Musella, 'Metahamiltonian groups and related topics', Int. J. Group Theory 2 (2013), 117-129.

[6] F. de Giovanni and M. Trombetti, 'Uncountable groups with restrictions on subgroups of large cardinality’, J. Algebra 447 (2016), 383-396.

[7] F. de Giovanni and M. Trombetti, 'Nilpotency in uncountable groups', J. Aust. Math. Soc. 103 (2017), 59-69.

[8] F. de Giovanni and M. Trombetti, 'The class of minimax groups is countably recognizable', Monatsh. Math. 185 (2018), 81-86.

[9] F. de Giovanni and M. Trombetti 'Groups with restrictions on proper uncountable subgroups', Studia Sci. Math. Hungar. 56 (2019), 154-165.

[10] F. de Giovanni and M. Trombetti, 'Pronormality in group theory', Adv. Group Theory Appl. 9 (2020), 123-149.

[11] F. de Giovanni and G. Vincenzi, 'Pronormality in infinite group theory', Math. Proc. R. Ir. Acad. 100A (2000), 189-203.

[12] M. R. Dixon, M. J. Evans and H. Smith, 'Groups with all proper subgroups soluble-by-finite rank', J. Algebra 289 (2005), 135-147.

[13] M. R. Dixon, M. J. Evans and H. Smith, 'Some countably recognizable classes of groups', J. Group Theory 10 (2007), 641-653.

[14] A. Ehrenfeucht and V. Faber, 'Do infinite nilpotent groups always have equipotent abelian subgroups?', Indag. Math. 34 (1972), 202-209. 
[15] D. Esposito, F. de Giovanni and M. Trombetti, 'Groups whose nonnormal subgroups are metahamiltonian', Bull. Aust. Math. Soc. 102 (2020), 96-103.

[16] M. Ferrara and M. Trombetti, 'A countable-type theorem for uncountable groups', Adv. Group Theory Appl. 3 (2017), 97-114.

[17] M. Ferrara and M. Trombetti, 'Groups whose non-permutable subgroups are metaquasihamiltonian', J. Group Theory 23 (2019), 513-529.

[18] M. Ferrara and M. Trombetti, 'A local study of group classes', Note Mat. 40(2) (2020), 1-20.

[19] M. Ferrara and M. Trombetti, 'Locally finite simple groups whose non-nilpotent subgroups are pronormal', Preprint.

[20] N. F. Kuzennyı and I. Y. Subbotin, 'Groups in which all of the subgroups are pronormal', Ukrainian Math. J. 39(3) (1987), 251-254.

[21] P. Longobardi, M. Maj and H. Smith, 'A note on locally graded groups', Rend. Semin. Mat. Univ. Padova 94 (1995), 275-277.

[22] A. Y. Ol'shanskii, Geometry of Defining Relations in Groups (Kluwer Academic, Dordrecht, 1991).

[23] G. Oman, 'Some results on Jónsson modules over a commutative ring', Houston J. Math. 38 (2010), 3489-3498.

[24] T. A. Peng, 'Finite groups with pro-normal subgroups', Proc. Amer. Math. Soc. 20 (1969), 232-234.

[25] D. J. S. Robinson, 'A theorem on finitely generated hyperabelian groups', Invent. Math. 10 (1970), $38-43$.

[26] D. J. S. Robinson, Finiteness Conditions and Generalized Soluble Groups (Springer, Berlin, 1972).

[27] D. J. S. Robinson, 'Groups whose homomorphic images have a transitive normality relation', Trans. Amer. Math. Soc. 176 (1973), 181-213.

[28] D. J. S. Robinson, 'Groups in which normality is a transitive relation', Proc. Cambridge Philos. Soc. 60 (1964), 21-38.

[29] J. S. Rose, 'Finite soluble groups with pronormal system normalizers', Proc. Lond. Math. Soc. (3) 17 (1967), 447-469.

MARIA FERRARA, Dipartimento di Matematica e Fisica,

Università degli Studi della Campania 'Luigi Vanvitelli',

Viale Lincoln 5, Caserta, Italy

e-mail: maria.ferrara1@unicampania.it

MARCO TROMBETTI, Dipartimento di Matematica e Applicazioni

'Renato Caccioppoli', Università degli Studi di Napoli Federico II,

Complesso Universitario Monte S. Angelo,

Via Cintia, Napoli, Italy

e-mail: marco.trombetti@unina.it 